\title{
Individual differences in information processing during concept learning'
}

\author{
LEONARD I. JACOBSON, JIM MILLHAM, and STEPHEN E. \\ BERGER, University of Miami, Coral Gables, Fla. 33124
}

Information processing strategies of $S$ s differing in intelligence were evaluated. Ss of lowest intelligence were found to use the same processes in learning both mediated and nonmediated concepts, whereas, high-intelligence Ss used symbolic processes in learning mediated concepts which they did not employ in learning nonmediated concepts. These findings suggest that mediation effects may not operate across all $S s$ as is often assumed.

Previous investigations have demonstrated that the relationship between intelligence and information processing during concept learning is a complex one that is contingent upon the specific measures of intelligence and concept learning employed and the use of a mediation or nonmediation paradigm (Jacobson, Dickinson, Fleishman, \& Haraguchi, 1969; Jacobson, Elenewski, Lordahl, \& Liroff, 1968). In a study employing the method of successive presentation (response method), it has been demonstrated also that vocabulary tests, which are heavily loaded with the primary intelligence factor, are excellent predictors of mediated, but not nonmediated, concept learning (Jacobson et al, in press, a). Finally, the effects of intelligence have been found to operate primarily during the period prior to the first correct response in mediated concept learning (Jacobson et al, in press, a, b).

In the successive-presentation task, mediated concept learning may be viewed as a complex form of verbal learning which permits considerable use of symbolic processes in the identification of commonalities among sets of elements. During the period preceding the first correct response, mediated concept learning constitutes a generative problem in which Ss scan storage units in memory in order to produce correct responses. It appears that the superiority of highly intelligent Ss resides in effective processing and retrieval of information, resulting in the generation of correct responses.

After this response-generation stage is completed, the problem becomes one in which Ss must generalize a correct response to related instances in a set of limited size. This phase may be termed the recognition stage of concept learning; performance during this period has been found to be unrelated to differences in intelligence.

The present study investigated a further question: Do individuals of varying intelligence use the same cognitive processes but with differential efficiency, or do they employ different processes in retrieving information in mediated conceptualization?

\section{METHOD}

The Ss were 115 undergraduate volunteers. Each $S$ learned one of two paired-associate lists and a concept list (Mednick \& Freedman, 1960). The first paired-associate list (PAL-1) was designed to facilitate the attainment of the concept "white" in subsequent concept learning. On this list, the learning of 4 of the 12 word pairs facilitated the attainment of "white" in later concept learning. The remaining word pairs on this list were neutral with regard to "white." The second paired-associate list (PAL-2) was similarly designed but facilitated the attainment of the concept "soft." The concept list consisted of 12 nouns which could be placed into three groups, each of which contained 4 nouns which were instances of a common concept. The Ss' task was to learn the three adjectives ("white," "soft," and buffer concept "large") each of which described a different group of 4 nouns. Mediation and nonmediation concept learning scores were obtained for each $\mathrm{S}$.

Ss learned PAL-1 or PAL-2 to a criterion of two successive perfect trials. They then learned the concept list to a criterion of one perfect performance or were terminated at 20 trials. The lists were presented at $4 \mathrm{sec}$ intervals and were projected on a screen by a Kodak Carousel Slide Projector connected to a time-interval generator. Three orders of each paired-associate list and five orders of the concept list were employed and kept constant for all Ss. All Ss were then administered the Vocabulary test of the Wechsler Adult Intelligence Scale.

\section{RESULTS}

Analysis of variance indicated that concepts were learned more readily under the mediation condition. Significant mediation effects were in evidence for the number of trials to the first correct response, the response-generation stage of concept learning $(p<.001)$, and for total errors $(p<.003)$. Significant effects of intelligence were found also for the number of trials required to complete the response-generation stage of concept learning $(p<.003)$, and for total errors $(p<.01)$. No significant effects of mediation or intelligence (ps $>.10)$ were found during the period from the first correct response to concept attainment, the recognition stage of concept learning.

The relationship between intelligence and the use of symbolic processes was then evaluated. Figure 1 indicates the number of trials necessary for Ss of high, medium, and lowest intelligence to complete the response-generation stage of mediated (M) and nonmediated (NM) concept learning. Ss of highest intelligence demonstrated a significant improvement in completing the response-generation stage in the mediation condition over performance in the nonmediation condition $(t=3.916, d f=78, p<.001)$. Ss of medium intelligence demonstrated a significant, but lesser, improvement in the mediation condition $(t=2.623, d f=80, p<.02)$. Ss of lowest intelligence demonstrated no significant improvement in

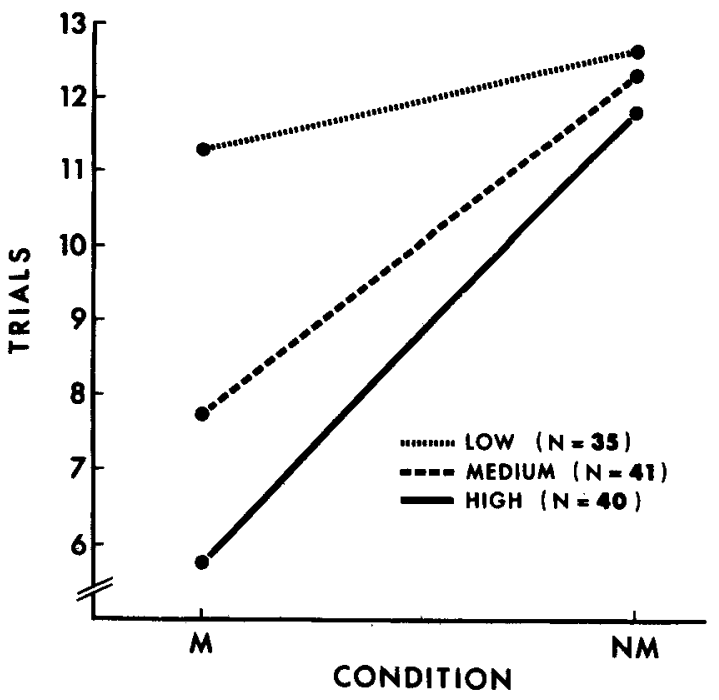

Fig. 1. Mean number of trials for Ss differing in intelligence to complete the response-generation stage of mediated and nonmediated concept learning.

(Continued on page 289) 
$F(8,102)=6.75, p>.25$, but was significant for Rote, $\mathrm{F}(8,102)=33.52, \mathrm{p}<.001$, and for Not-the-Same NLM, $F(8,102)=39.48, p<.001]$. The difference between Rote and Not-the-Same NLM was negligible after 1 day, and, after 3 days and 9 days, retention for Not-the-Same tended to be lower though not significantly [Scheffé values of $F(8,102)=8.80, p>.25$ and $F(8,102)=10.88, p>.25$, respectively]. Not only did forgetting of items with Not-the-Same NLM increase with increasing retention interval, but also the frequency of Not-the-Same NLMs increased with longer retention intervals $(4.9 \%$ of all items for the 1-day group had Not-the-Same NLM, 9.9\% for the 3-day group, and $22.7 \%$ for the 9-day group).

\section{DISCUSSION}

The results of the present study supported previous investigations showing that the method a $S$ uses in learning paired associates is highly related to retention. In particular, the findings of Montague et al (1966) and Adams \& McIntyre (1967) were confirmed and extended. In the present study, the facilitative effect of NLMs was found to extend over a retention interval as long as 9 days. While the recall of pairs learned by rote or with an NLM that was forgotten at recall became increasingly poor with longer retention intervals, the pairs with the NLMs available at recall were correctly remembered over $90 \%$ of the time even after 9 days.

Natural language mediation may be thought of as an instance of transfer of training (Adams, 1967, pp. 86-88; Adams \& Montague, 1967). According to this view, the $S$ has a long history of using language, and when faced with the task of learning a pair of items he may try to integrate the pair with natural language habits already in his response repetoire. Thus, a high degree of associative strength is quickly achieved through its integration with existing language habits. Obviously, a S may not always have the ability or desire to integrate an item with his past language habits. In such cases rote learning is used. This is in no way incompatible with the view that learning can occur through the association of new items with old language habits.

The transfer of training hypothesis carried with it, implicitly, the explanation of reduced forgetting for mediated items. NLMs, representing the transfer of established habits to the new learning task, impart associative strength to the new material that decreases forgetting. Furthermore, mediated items are an NLM-item complex that has a relative uniqueness with respect to other language units and the chances of forgetting through interference theory of forgetting, but this seems reasonable because it is the most defensible theory of forgetting presently available (Adams, 1967).

\section{REFERENCES}

ADAMS, J. A. Human memory. New York: McGraw-Hill, 1967.

ADAMS, J. A., \& McINTYRE, J. S. Natural language mediation and all-or-none learning. Canadian Journal of Psychology, 1967, 21 , 436-449.

ADAMS, J. A., \& MONTAGUE, W. E. Retroactive inhibition and natural language mediation. Journal of Verbal Learning \& Verbal Behavior, $1967,6,528-535$.

BATTIG, W. F. Procedural problems in paired-associate learning research. Psychonomic Monograph Supplements, 1965, 1 (Whole No. 1).

MONTAGUE, W. E., ADAMS, J. A., \& KIESS, H. O. Forgetting and natural language mediation. Journal of Experimental Psychology, $1966,72,829-833$.

MONTAGUE, W. E., \& KIESS, H. O. The associability of CVC pairs. Journal of Experimental Psychology, 1968, 78, (Monograph Supplement, 2, Part 2).

REED, H. B. Associative aids: I. Their relation to learning, retention and other associations. Psychological Review, 1918, 25, 128-155.

\section{NOTE}

1. This report is based on a thesis submitted to the Graduate College of the University of Illinois in partial fulfillment of the requirement for the M.A. degree in psychology. The author wishes to thank Drs. Jack A. Adams and William E. Montague for their helpful advice and encouragement.

\section{(Continued from page 287)}

mediated concept learning over their performance in nonmediated concept learning $(t=0.684$, $d f=68)$. It may be concluded that highly intelligent $S s$ effectively used the information provided by the mediation condition, whereas Ss of lowest intelligence were not helped by the additional input.

Similar findings were in evidence for highest and lowest intelligence Ss when the performance measure was total errors. Significantly fewer errors in mediated concept learning were found for $S s$ of highest intelligence $(t=3.074$, $\mathrm{df}=78$, $p<.01)$, but not for Ss of lowest intelligence $(t=1.114$, $\mathrm{df}=68$ ). In contrast to the significant improvement in performance noted above for the trials measure, no significant reduction in errors was found during mediated concept learning for Ss of medium intelligence $(t=1.473, \mathrm{df}=80)$.

\section{DISCUSSION}

These findings indicate that $S$ s of high intelligence solve mediation problems by the use of symbolic processes which result in efficient information retrieval and problem solution. In contrast, Ss of lowest intelligence use the same processes for retrieving information in mediated concept learning that they employ in nonmediated learning. As a result, the Ss of lowest intelligence in the present experiment demonstrated no significant improvement in performance in mediated concept learning. The lesser effect found for the medium intelligence
Ss may have resulted from combining within this group scores of Ss who were similar to the highest intelligence group and others who were similar to the lowest intelligence group.

These data also provide information concerning the role of mediation in learning. A general mediation effect was found but further analysis indicated that this effect operated only for a subset of the Ss. The demonstration that mediation occurred for Ss of highest but not of lowest intelligence emphasizes the interaction of mediation conditions and individual differences. These findings suggest that mediation effects may not operate across all Ss as is often assumed.

\section{REFERENCES}

JACOBSON, L. I., DICKINSON, T. C., FLEISHMAN, J. M., \& HARAGUCHI, R. S. The relationship of intelligence and mediating processes to concept learning. Journal of Educational Psychology, in press, a.

JACOBSON, L. I., ELENEWSKI, J. J., LORDAHL, D. S., \& LIROFF, J. $H$. The role of creativity and intelligence in conceptualization. Journal of Personality \& Social Psychology, in press, $b$.

MEDNICK, S. A., \& FREEDMAN, J. L. Facilitation of concept formation through mediated generalization. Journal of Experimental Psychology, 1960, 60, 278-283.

\section{NOTE}

1. This research was supported by Research Grant MH14716-01 from the National Institute of Mental Health. We wish to express our thanks fo Neil Schneiderman for his assistance. 